\title{
Zur Kenntnis der isomeren Dinitrobenzole
}

\section{Doctoral Thesis}

Author(s):

Wyler, Otto

Publication date:

1932

Permanent link:

https://doi.org/10.3929/ethz-a-000104565

Rights / license:

In Copyright - Non-Commercial Use Permitted 


\section{Zur Kenntnis der isomeren Dinitrobenzole}

Von der

Eidgenössischen Technischen Hochschule in Zürich zur Erlangung der

Würde eines Doktors der technischen Wissenschaften genehmigte

Promotionsarbeit

vorgelegt von

\section{Otto Wyler}

Dipl. Ingenieur-Chemiker aus Ober-Endingen (Aargau)

Nr. 679

Referent: Herr Prof. Dr. H. E. Fierz

Korreferent: Herr Prof. Dr. L. Ruzicka

\section{BASEL}

Buchdruckerei Emil Birkhäuser \& Cie.

1932 
grund stehen, wobei am Schlusse der Reaktion der Alkohol direkt abdestilliert werden kann. Durch die Vakuumdestillation dürfte ein 99,6-proz. Produkt erhalten werden.

Zum Schlusse sei noch ein kleiner Beitrag zur physiologischen Wirkung der Dinitrobenzole gegeben. Der Schreibende war in der glücklichen Lage, gegen das Dinitrobenzol als solches in grossem Masse unempfindlich zu sein. Die direkte häufige Berührung des Dinitrobenzols übte absolut keine nachteilige Wirkung aus. Hingegen zeigte es sich, dass die eingeatmeten Dämpfe, insbesondere die Äthylalkohol-haltigen (im Gegensatz dazu bei Methylalkohol kaum), die üblichen Vergiftungserscheinungen (akute Cyanose) hervorriefen, allerdings nicht in dem in der Literatur beschriebenen ${ }^{1}$ ) Masse. Die äusserlichen Symptome verschwanden im allgemeinen wieder nach einem guten Schlafe. Kopfweh konnte nur in einem besonders krassen Falle beobachtet werden. Eine Blutuntersuchung 6 Tage nach dieser Vergiftung zeigte hingegen eine normale Zahl von Erythrocythen und keine Degenerationserscheinung des Blutes. Immerhin ist trotzdem bei jedem Arbeiten mit Dinitrobenzol äusserste Sorgfalt und vor allem Reinlichkeit am Platze. - Die drei Dinitrobenzole untereinander zeigen, analog ihrem chemischen Verhalten, in ihrer Giftigkeit keine grossen Unterschiede. Das p-Dinitrobenzol scheint etwas heftiger zu wirken, als die beiden andern.

\section{Zusammenfassung.}

1. In den vorstehenden Untersuchungen wurde bestätigt, dass bei der Nitrierung des Nitrobenzols stets alle drei Dinitrobenzole gebildet werden. Der Gehalt an m-Dinitrobenzol richtet sich nach der Nitriertemperatur, jedoch schwankt er nur innerhalb der verhältnismässig kleinen Grenzen von $85-95 \%$. Um ein reineres m-Dinitrobenzol zu gewinnen, ist daher nach jeder Nitrierung eine Nachbehandlung notwendig. - Durch Katalysatoren können keine besseren Resultate erzielt werden.

2. Das Verhältnis von ortho- zu para-Dinitrobenzol verschiebt sich bei steigender Nitrierungstemperatur von $51: 49 \%\left(-17^{\circ}\right)$ auf $97: 3 \%\left(+129^{\circ}\right)$.

3. Die Angaben von Holleman und de Bruyn wurden durch die vorliegende Arbeit bestätigt und erweitert.

4. Die ähnlichen physikalischen und chemischen Eigenschaften erschweren eine Trennung der Isomeren. Immerhin gelingt es, unter merklichen Verlusten an m-Dinitrobenzol, dieses durch Umkrystalli-

1) Ztschr. ges. Schiess- u. Sprengstoffw. 13, 245, 268 (1918); 14, 143 (1919). 
sieren in Methyl- oder Äthylalkohol rein zu gewinnen. Besser aber ist die chemische Trennung. Hier ist vor allem die Reaktion 'mit Natriumdisulfid technisch verwendbar, unter Anwendung von heissem Methyl- oder Äthylalkohol als Lösungsmittel und nachheriger Vakuumdestillation des unzersetzten Dinitrobenzols. Hierbei wird ein nahezu chemisch reines m-Dinitrobenzol in guter Ausbeute erhalten. - Für wissenschaftliche Zwecke empfiehlt es sich hingegen, mit Natriumhydroxyd, Natriummethylat oder Ammoniak und Methylalkohol als Lösungsmittel zu arbeiten. Die Gesamtausbeute bleibt indes hinter derjenigen mit Disulfid zurück. - Bei allen diesen alkalischen Reinigungsmethoden schadet aber die Gegenwart von Wasser ganz beträchtlich, indem dadurch auch das m-Dinitrobenzol mitangegriffen wird, am meisten wohl bei methylalkoholischem Ammoniak.

5. Zur Ermittlung des Isomerengehalts der Dinitrobenzole dient eine kombinierte chemisch-physikalische Analyse. Für die chemische Analyse dient die Reaktion mit Natriummethylat zur Bestimmung der Summe von o- und p-Dinitrobenzol, wobei eine ihnen äquivalente Menge Methylat verbraucht wird. Daraus kann der Gehalt an m-Dinitrobenzol, als Ergänzung zu 100\%, in guter Annäherung ermittelt werden. Zur Bestimmung der o- und p-Isomeren bedient man sich, als physikalische Analyse, des Schmelzpunktes des im Vakuum destillierten Dinitrobenzols, unter Zuhilfenahme des Schmelzdiagramms der Dinitrobenzole. - Auf diese Weise lassen sich alle Gemische der drei isomeren Dinitrobenzole in befriedigender Weise bestimmen. 